play a part in the important general question of charge motion and storage in wide-band solids.

\section{Planetary collisions}

\section{from David $W$. Hughes}

At the dawn of the Solar System collisions between planets could have been so frequent and devastating that we now see only the scattered remnants of this scrummage. Interaction between planets in their present orbits is minimal, a situation which slowly built up as the Solar System aged. But small bodies, such as comets and asteroids, often have orbits which cross those of the planets and thus are intrinsically unstable. They may be eliminated by collision with a planet-either accreting to the planetary surface or (more rarely) being fragmented when they graze the planet's upper atmosphereor they may be perturbed-either into shorter period orbits with aphelia near the planet's orbit (like the Jovian family of comets) or into hyperbolic orbits which take them out of the Solar System (like the space probes Pioneer 10 and 11).

Probabilities of encounter were first calculated in a classic series of papers by Öpik. Two fundamental approaches can be made to the problem, the analytical one which considers individual particles and their orbital elements and the numerical integration approach which uses random walk and Monte Carlo techniques applied to a large number of particles. These two approaches have been combined by Weidenschilling in a recent paper in The Astronomical Journal (80, 145; 1975). The probability of encounter between two objects with intersecting orbits is found to be simply a function of their relative velocities and one angular variable. These encounters will eventually occur even between particles with crossing orbits (orbits with the same range of heliocentric distances) because secular perturbations cause nodal precession and advancement of the perihelion, which finally results in intersections and then encounters.

Öpik (Adv. Astron. Astrophys., 2, 219 ; 1963) found that the mean lifetime of the Apollo group of asteroids (which have orbits crossing those of Earth and Mars) is $10^{8}$ years. Collisions would have caused their numbers to decay exponentially with time. So if these asteroids are remnants of an original group formed 4,500 $\mathrm{Myr}$ ago at the birth of the Solar System, there would have been $2.5 \times 10^{19}$ of them at $t=0$, equivalent to a mass one hundred times that of the Sun. Clearly this is most unlikely, and thus the asteroids in this group cannot be permanent members of the Solar System but must be continually replenished from the asteroidal belts and comets.

Weidenschilling comments on the differences in the encounter probability curves of the terrestrial and Jovian planets. This is partly explained by the great mass of Jupiter making it more likely than the inner planets to encounter material. But the mass of the planet also affects the ultimate fate of the small bodies encountered. The terrestrial planets, with orbital velocities several times greater than their escape velocities, have effective cross sections such that the ejection probability is only an order of magnitude higher than the collision probability. For Jupiter the orbital velocity is less than the escape velocity, the collision cross section varies with Joviocentric velocity and about 1,000 more particles will be ejected from the Solar System by Jupiter than collide with it.

Weidenschilling's figure for the probability of ejection by Jupiter is an order of magnitude greater than Öpik's whereas his results for the terrestrial planets agree with those of Öpik. If Weidenschilling's Jupiter result is correct, the time scale for the dynamical elimination of comets must be two to four orders of magnitude greater than the time scale for disintegration. Thus the fact that thousands of dead cometary nuclei are not observed indicates that most comets disintegrate completely or that the dead nuclei are too small to be seen.

The equations given in Weidenschilling's paper also enable the efficiency of cometary ejection processes to be calculated. The author promises to apply these to the production of the Oort cloud of distant comets in a later paper. He also speculates that the total mass and angular momentum lost from the Solar System by cometary ejection might be of cosmogonical significance, indicating that the Solar System's angular momentum-mass balance might have varied with time.

\title{
Type B hepatitis a kissing disease?
}

\section{from Arie J. Zuckerman}

THough the importance of the parenteral and inapparent parenteral route of transmission of hepatitis B infection has long been recognised, it has become difficult to incriminate parenteral transmission in all cases of infection with hepatitis $\mathbf{B}$ virus (Tech. Rep. Ser. No. 570; World Health Organisation, 1975). Interest in various mechanisms of spread of this infection has flourished in recenit years following the experimental demonstration of infectivity of serum by the oral route and epidemiological studies implying the possibility of venereal transmission of hepatitis B (Nature, 246, 59; 1973). Although the reported detection of hepatitis B surface antigen in semen and menstrual blood obtained from individuals with antigenaemia offers possible explanations for infection by the sexual route, the matter is far from settled.

The presence of hepatitis B surface antigen in a variety of body fluids and excretions such as urine, colostrum, milk, saliva, bile, faeces and sweat has not been consistently confirmed. In particular confirmation of the finding of the antigen in bile and faeces is still lacking in spite of intensive studies in various laboratories.

A recent report from Costa Rica has shed some light on possible nonparenteral noutes of transmission of hepatitis B in endemic settings (Villarejos et al., New Engl. J. Med.,
291, 1375; 1974). Serial samples of faeces, urine and saliva collected from chronic carriers of hepatitis B surface antigen and from patients with acute hepatitis B were tested by radioimmunoassay. The antigen was not found in any of 120 faecal extracts examined. The antigen was detected in three of 130 urine samples and all the positive samples contained occult blood. 274 saliva specimens from 93 chronic carriers were tested and the antigen was found in nearly $60 \%$ of specimens belonging to 75 subjects. It is interesting to note that the antigen was not found constantly in the saliva of the carriers, although all had circulating antigen at the time of sampling. Antigen was found in the saliva of $76 \%$ of 41 patients with acute hepatitis $B$, mainly during the first three weeks after the onset of clinical symptoms. The presence of the surface antigen in saliva both in carriers and in patients with the acute disease was not related to contamination with blood. These results show that salivary transmission may be a major mechanism of spread of infection in an area where hepatitis $B$ occurs endemically. Villarejos and colleagues conclude therefore that transmission may occur directly by mouth-to-mouth kissing and in children by exchange of chewed toys and sweets. This presupposes of course that the antigen found in saliva is actually infective. 